\title{
A new constitutively active mutant of AMP-activated protein kinase inhibits anoxia-induced apoptosis of vascular endothelial cell
}

\begin{abstract}
Daisuke Nagata ${ }^{1,2}$, Arihiro Kiyosue ${ }^{2}$, Masao Takahashi $^{2}$, Hiroshi Satonaka ${ }^{3}$, Kimie Tanaka ${ }^{1,2}$, Masataka Sata ${ }^{2}$, Tetsuo Nagano ${ }^{4}$, Ryozo Nagai ${ }^{2}$ and Yasunobu Hirata ${ }^{2}$

The inhibition of apoptotic changes in vascular endothelial cells is important for preventing vascular damage from hypoxia. AMP-activated protein kinase (AMPK) has recently been identified as playing a role in vascular protection. Although the chemical reagent 5-aminoimidazole-4-carboxamide-1- $\beta$-D-ribofuranoside (AICAR) has been used to stimulate AMPK activity, AICAR has been associated with several nonspecific reactions. We therefore constructed a new constitutively active mutant of AMPK $\alpha 1$ (NcaAMPK), which lacks the autoinhibitory domain in AMPK $\alpha 1$ and in which threonine 172 has been replaced with aspartate. We investigated whether NcaAMPK has an anti-apoptotic effect in vascular endothelial cells under anoxic conditions. NcaAMPK, or green fluorescent protein (GFP) as a control, was overexpressed in human umbilical vein endothelial cells (HUVECs). After HUVECs were incubated for $40 \mathrm{~h}$ under normoxic or anoxic conditions, we examined cell viability, caspase $3 / 7$ activity, and expression and phosphorylation levels of apoptosis-related proteins. Cell viabilities under anoxic conditions were improved in NcaAMPK-overexpressing cells. Anoxia increased caspase 3/7 activity, but NcaAMPK reduced this increase significantly. NcaAMPK overexpression increased protein kinase B/Akt Ser473 and endothelial nitric oxide synthase Ser1177 phosphorylation, but pretreatment with the nitric oxide synthase inhibitor $N^{G}$-nitro-L-arginine methyl ester (L-NAME) did not decrease the viability of NcaAMPK-overexpressing HUVECs. Furthermore, co-expression of a dominant-negative Akt reduced the improvement in cell viability and the suppression of poly (ADP-ribose) polymerase cleavage by NcaAMPK under anoxic conditions. In conclusion, NcaAMPK inhibited anoxia-induced apoptosis in vascular endothelial cells through Akt activation, suggesting that activation of AMPK might protect against ischemic vascular injury.

Hypertension Research (2009) 32, 133-139; doi:10.1038/hr.2008.25; published online 23 January 2009
\end{abstract}

Keywords: Akt; AMP-activated protein kinase; anoxia; apoptosis; vascular endothelial cell

\section{INTRODUCTION}

An important initial step in the pathogenesis of atherosclerosis is endothelial damage by various factors, such as inflammatory cytokines. ${ }^{1}$ A large body of evidence has shown that hypoxia is a pivotal factor modulating endothelial cell function and survival. ${ }^{2}$ Although necrosis is a major pathway for regulating ischemia- and reperfusioninduced cardiomyocyte death, apoptosis has recently been identified as another important regulator. ${ }^{3}$ Furthermore, apoptosis of vascular endothelial cells is thought to be a pivotal regulator of vascular damage, suggesting that apoptosis of endothelial cells could be an important therapeutic target for preventing cardiovascular diseases.

AMP-activated protein kinase (AMPK) has been identified as a key regulator of cellular ATP levels. ${ }^{4}$ AMPK was identified as a homolog of yeast sucrose non-fermenting 1 and is known as a metabolite-sensing protein kinase. ${ }^{5}$ AMPK is a heterotrimeric serine/threonine protein kinase consisting of a catalytic $\alpha$-subunit and two regulatory subunits, $\beta$ and $\gamma .{ }^{6}$ There are multiple isoforms of each AMPK subunit, with $\alpha 1$, $\alpha 2, \beta 1, \beta 2, \gamma 1, \gamma 2$, and $\gamma 3$ forming heterotrimers ${ }^{7}$ that differ in tissue and subcellular localization. In mammalian cells, AMPK is activated by increases in the AMP/ATP ratio, ${ }^{4}$ which occur under conditions of hypoxia or anoxia. ${ }^{8,9}$ When the AMP/ATP ratio increases, AMPK is partially activated by a conformational change after combining with AMP and is fully activated when phosphorylated at Thr172 by an AMPK kinase (AMPKK), which is now known to be LKB1 serine/ threonine kinase. ${ }^{10}$ Activated AMPK phosphorylates and downregulates several anabolic enzymes, including 3-hydroxy-3-methylglutarylCoA reductase or acetyl-CoA carboxylase, and shuts off the ATPconsuming synthetic pathway. ${ }^{4}$ In addition to such energy-saving

${ }^{1}$ Department of Molecular Research for Vascular Diseases, Graduate School of Medicine, University of Tokyo, Tokyo, Japan; ${ }^{2}$ Department of Cardiovascular Medicine, Graduate School of Medicine, University of Tokyo, Tokyo, Japan; ${ }^{3}$ Department of Nephrology and Endocrinology, Graduate School of Medicine, University of Tokyo, Tokyo, Japan and ${ }^{4}$ Department of Chemistry and Biology, Graduate School of Pharmaceutical Sciences, University of Tokyo, Tokyo, Japan

Correspondence: Dr D Nagata, Department of Molecular Research for Vascular Diseases, Graduate School of Medicine, University of Tokyo, 7-3-1 Hongo, Bunkyo-ku, Tokyo 1138655, Japan.

E-mail: dskngtendo0504-tky@umin.ac.jp

Received 18 August 2008; revised 7 October 2008; accepted 27 October 2008; published online 23 January 2009 
effects, AMPK is important for protecting cellular function under energy-restricted conditions, such as hypoxia/anoxia. ${ }^{11}$

To investigate the functions of AMPK in mammalian cells, we and other investigators have used 5-aminoimidazole-4-carboxamide-1- $\beta$ D-ribofuranoside (AICAR), which is a chemical AMPK activator. AICAR is phosphorylated and converted to 5-aminoimidazole-4carboxamide-1- $\beta$-D-ribofuranosyl-5'-monophosphate (ZMP) in mammalian cells. ZMP mimics the effect of AMP and activates AMPK. ${ }^{12,13}$ However, AICAR increases not only ZMP but also $\mathrm{ZTP}^{12}$ and, as a chemical rather than a specific enzyme, may have other nonspecific effects on ATP-requiring reactions. Constitutively active mutants of AMPK provide a more specific method for examining the functions of AMPK. Several kinds of constitutively active AMPK mutants have been investigated, including AMPK $\alpha 1$ (amino acids (aa) 1-312, Thr172 $\rightarrow$ Asp: T172D) ${ }^{14,15}$ AMPK $\gamma 2($ Arg302 $\rightarrow$ Gln), ${ }^{16}$ and AMPK $\gamma 1$ (His150 $\rightarrow$ Arg).${ }^{17}$ Although it has been reported that maximum activity of AMPK requires all three subunits, ${ }^{6}$ AMPK $\alpha 1$ (1-312, T172D) lacks both the binding domain for interactions with the $\beta$-subunit and the autoinhibitory domain, which inhibits the self-kinase activity. ${ }^{14}$ The $\beta$-subunit plays a role in modulating subcellular localization through its phosphorylation and myristoylation, ${ }^{18,19}$ so that the ability to bind the $\beta$-subunit might be critical for the catalytic $\alpha$-subunit to select its appropriate substrates. We have constructed a replication-defective adenoviral vector expressing a new constitutively active AMPK $\alpha 1$ mutant (NcaAMPK), which lacks the autoinhibitory domain (aa 313-392) but has the complex formation domain (aa 393-548). ${ }^{14}$ As we found that NcaAMPK functions as a specific and continuous activator of AMPK, we investigated whether NcaAMPK overexpression could inhibit the pro-apoptotic pathway induced by anoxia in human umbilical vein endothelial cells (HUVECs).

\section{MATERIALS AND METHODS}

\section{Chemical reagents}

$N^{\mathrm{G}}$-nitro-L-arginine methyl ester (L-NAME), wortmannin, and other chemical reagents were purchased from Sigma-Aldrich (St Louis, MO, USA).

\section{Antibodies}

AMPK $\alpha 1$, phospho-AMPK $\alpha$ (Thr172), phospho-protein kinase B/Akt (Ser473), phospho-endothelial nitric oxide synthase (eNOS) (Ser1177), and poly (ADP-ribose) polymerase (PARP) antibodies were purchased from Cell Signaling Technology (Beverly, MA, USA). Actin, Akt, and eNOS antibodies were purchased from Santa Cruz Biotechnology (Santa Cruz, CA, USA). Myc-tag and hemagglutinin (HA)-tag antibodies were purchased from Upstate Biotechnology (Lake Placid, NY, USA).

\section{Cell culture and anoxic conditions}

HUVECs were purchased from Kurabo (Osaka, Japan) and cultured in HuMedia EG2 (Kurabo). HUVECs were used for experiments within passages 6-8. In some experiments, HUVECs were transduced with the indicated replication-defective adenoviral vectors at a multiplicity of infection of 50 plaque-forming units (50 MOI) for 1 day. The medium was then changed to a low-serum medium, HuMedia basic medium (EB2) with $0.2 \%$ fetal bovine serum, to reduce the effects of stimulation by serum mitogens.

After incubation in the low-serum medium for $8 \mathrm{~h}$, adenovirus-infected cells were incubated in a normoxic or anoxic incubator for $40 \mathrm{~h}$. In the pilot experiments, we found that $24 \mathrm{~h}$ anoxia was too short to evaluate cell viability because cell survival rates under these conditions were $>90 \%$. Furthermore, even under normoxic conditions, $>72 \mathrm{~h}$ incubation in the low-serum medium induced high rates of cell death $>40 \%$. We conclude that $40-48 \mathrm{~h}$ anoxic conditions are suitable for evaluating effects of anoxia on cell death without the cell-damaging effects of low serum. For anoxic conditions, we used the Anoxic Chamber System (Coy Laboratory Products, Grass Lake, MI, USA). To exclude the effects of reoxygenation after anoxia, every procedure (such as protein extraction) was performed under anoxic conditions in this anoxic chamber.

\section{Construction of adenoviral AMPK $\alpha 1$ mutant vectors and other adenoviral vectors}

Total RNA was extracted from rat vascular smooth muscle cells (rVSMCs) using Trizol reagent (Invitrogen, Carlsbad, CA, USA) according to the manufacturer's instructions. cDNA was produced by reverse transcription from purified rVSMC RNA with an RNA LA PCR kit (TAKARA, Otsu, Japan) using a random 9mer primer. Synthetic oligo primers for PCR and for creating point mutations are as follows:

primer 1

GGAATTCGCCATGGAGCAGAAGCTTATCTCCGAGGAGGACCTCGGTG GCGGCGAGAAGCAGAAGCACGACGGG

primer 2

CCGCTCGAGTTAGTACAGGCAGCTGAGGACCTC

primer 3

GCTCTAGAGTACAGGCAGCTGAGGACCTC

primer 4

GCTCTAGAAAGGCAAAGTGGCATTTGGGGATTCGAA

primer 5

GCTCTAGAGCATGCTCGAGTTACTGTGCAAGAATTTTAATTAGATTTG CACACACATTTCA

primer 6

ATGTCAGATGGTGAATTTTTAAGAGATAGCTGTGGCTCGCCCAATTATG primer 7

CATAATTGGGCGAGCCACAGCTATCTCTTAAAAATTCACCATCTGACAT

To make a conventional caAMPK (CcaAMPK) cDNA fragment that lacks aa 313-548, we performed PCR with KOD FX DNA polymerase (Toyobo, Osaka, Japan) using the rVSMC cDNA, primer 1 (which includes a myc-tag sequence), and primer 2. This fragment was digested with the restriction enzymes EcoRI and $\mathrm{XhoI}$ and ligated to pcDNA 3.1/Zeo(+) (Invitrogen) digested with EcoRI/ XhoI. To make a Thr-to-Asp mutation at residue 172, we used a QuickChange II XL Site-Directed Mutagenesis Kit (Stratagene, La Jolla, CA, USA) according to the manufacturer's instructions, using primers 6 and 7 to introduce the point mutation. To construct the NcaAMPK cDNA fragment, which has the complex formation domain but lacks the autoinhibitory domain, we amplified a fragment A using primer 1/primer 3 and a fragment B using primer 4/primer 5. We digested fragments $\mathrm{A}$ and $\mathrm{B}$ with $E c o \mathrm{RI} / \mathrm{Xba \textrm {I }}$ and $\mathrm{Xba \textrm {I }}$, respectively. Fragment A was then ligated to pcDNA 3.1/Zeo(+) digested with EcoRI/XbaI. Next, this vector was digested with $X b a \mathrm{I}$ and ligated with $\mathrm{XbaI}$-digested fragment $\mathrm{B}$. The direction of fragment $\mathrm{B}$ was confirmed by direct sequencing. To make a Thr-to-Asp mutation at residue 172, we used the QuickChange II XL Site-Directed Mutagenesis Kit, as for CcaAMPK construction. Schematics of the complete constructs are shown in Figure 1. We used an adenoviral construction kit (AdEasy adenovirus vector system; Stratagene) to make replication-defective adenoviral vectors for CcaAMPK and NcaAMPK. Briefly, pcDNA 3.1/Zeo $(+)$ plasmid vectors for CcaAMPK and NcaAMPK were digested with $K p n \mathrm{I} / \mathrm{XhoI}$, and the fragments were ligated to a KpnI/XhoIdigested pAdTrack-CMV plasmid, which was a generous gift from Dr Walsh (Boston University). We followed the manufacturer's instructions after this procedure. An adenoviral vector expressing green fluorescent protein (GFP) was obtained from Qbiogene (Illkirch, France) and used as a control for infection level. The HA-tagged dominant-negative (dn) Akt adenovirus was also generous gift from Dr Walsh.

\section{AMPK assay}

The AMPK assay using SAMS peptide (HMRSAMSGLHLVKRR, Upstate Biotechnology) was performed according to the methods available at the Animal Model of Diabetic Complications Consortium (AMDCC) website (http://www.amdcc.org/shared/phenotype/showAssay.aspx?id=260).

\section{Western blot analysis}

Western blot analysis was carried out as previously described. ${ }^{9}$ An ECL-PLUS Western Blotting Detection kit (GE Healthcare, Piscataway, NJ, USA) was used 


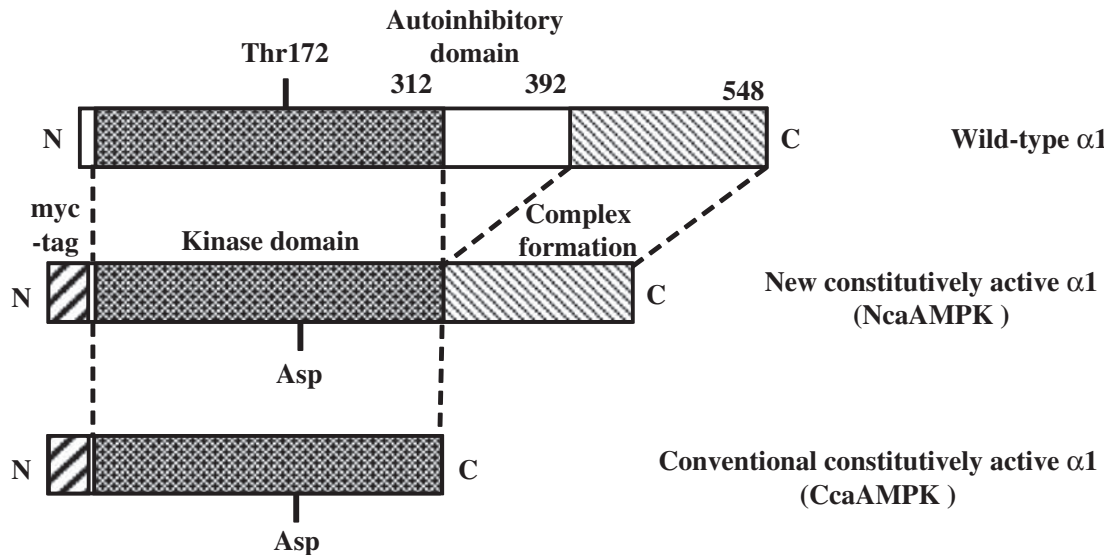

Figure 1 The construction of two AMPK $\alpha 1$ mutants, conventional constitutively active $\alpha 1$ (CcaAMPK) and new constitutively active $\alpha 1$ (NcaAMPK). Wildtype rat AMPK $\alpha 1$ contains a kinase domain, autoinhibitory domain, and the domain for complex formation. The autoinhibitory domain inhibits the AMPK $\alpha 1$ kinase activity. CcaAMPK lacks both the autoinhibitory domain and the domain for complex formation. At the target phosphorylation site of upstream kinases in the kinase domain of CcaAMPK, Asp was substituted for Thr172 to mimic phosphorylation. We constructed NcaAMPK by adding the domain for complex formation to the $\mathrm{C}$ terminus of CcaAMPK. Both CcaAMPK and NcaAMPK have a myc tag to distinguish their expression from that of endogenous AMPK $\alpha 1$.

for detection. The density of the bands was quantified using the Scion Image program (Scion Corporation, Frederick, MD, USA). Each experiment was repeated 3-4 times.

\section{Intracellular protein crosslinking experiments using photoactivated amino acids}

To evaluate intracellular AMPK protein complex formation in NcaAMPKoverexpressing cells, we used the photoactivated amino acid system (Thermo Fisher Scientific, Rockford, IL, USA) according to the manufacturer's instructions. Briefly, ultraviolet (UV)-sensitive L-photo-leucine and L-photo-methionine, which are incorporated in AMPK subunits or overexpressed AMPK mutants, are crosslinked to each other intracellularly after UV irradiation. Cell lysates were then extracted with cell lysis buffer, and AMPK protein complex formation was evaluated by western blotting.

\section{WST-1 assay}

To evaluate cell viability, we performed an assay using the WST-1 reagent (Roche, Basel, Switzerland) according to the manufacturer's instructions.

\section{Caspase $3 / 7$ assay}

We performed a caspase 3/7 assay using a Caspase-Glo 3/7 assay kit and GloMax96 luminometer (Promega, Madison, WI, USA) according to the manufacturer's instructions.

\section{Statistical analyses}

Values are expressed as the mean \pm s.e.m. Statistical comparisons were performed using analysis of variance with Scheffe's F procedure for post hoc analyses. $P$-value $<0.05$ was considered to be statistically significant.

\section{RESULTS}

NcaAMPK shows higher kinase activity and forms an AMPK complex more efficiently than CcaAMPK

To compare the AMPK activity of NcaAMPK and CcaAMPK, we performed a kinase assay by measuring the radiolabeled phosphorylation rate of SAMS peptide corrected by the amount of protein (Figure 2a). The basal levels of AMPK activity in GFP-overexpressing control cells was $1030 \pm 140$ c.p.m. per $\mu$ g protein. Although the kinase activity of CcaAMPK-overexpressing cells $(1860 \pm 160$ c.p.m. per $\mu \mathrm{g}$ protein) was significantly higher than that of controls $(P<0.05)$, the activity of NcaAMPK-overexpressing cells $(3310 \pm 250$ c.p.m. per $\mu \mathrm{g}$ protein) was significantly higher than that of CcaAMPK $(P<0.01)$.
We confirmed the expression of the CcaAMPK and NcaAMPK mutants by western blot (Figure $2 b$ ).

As NcaAMPK kinase activity is higher than CcaAMPK, we hypothesized that NcaAMPK would bind more effectively to the $\beta$-subunit than CcaAMPK and its kinase activity would be intensified. To show the formation of AMPK complexes including NcaAMPK, we used the UV light-sensitive photoactivated amino acid system. In NcaAMPKoverexpressing HUVECs, positive bands of approximately $130 \mathrm{kDa}$ were found when using an anti-myc-tag or anti-AMPK $\beta$ antibody (Figure 2c). In CcaAMPK-overexpressing cells, we did not find myctag positive bands of approximately $110 \mathrm{kDa}$, which indicates AMPK complex formation including the CcaAMPK mutant. When using the AMPK $\beta$ antibody, we found weaker intensity bands with a molecular weight of $\sim 135 \mathrm{kDa}$ in the GFP- and CcaAMPK-overexpressing cells. These might be endogenous AMPK complexes with expected molecular weights of $137 \mathrm{kDa}(\alpha 1 \beta 1 \gamma 1$ or $\alpha 2 \beta 1 \gamma 1)$. These results suggest that NcaAMPK, which has the complex formation domain, can form active AMPK complexes more effectively than CcaAMPK.

NcaAMPK overexpression inhibits anoxia-induced cell death We performed a WST-1 assay in HUVECs overexpressing GFP, CcaAMPK, or NcaAMPK to compare the effect of the AMPK mutants on cell survival under anoxic conditions. Cell viability was significantly higher $(P<0.01)$ in NcaAMPK-overexpressing cells than in either GFP or CcaAMPK-infected cells (Figure 3a). The lower panels in Figure 3a show phase-contrast micrographs of these cells. NcaAMPK overexpression inhibited cell death and kept cells attached to the bottom of the culture dish.

We also measured caspase $3 / 7$ activity to investigate whether anoxia-induced cell death might be accompanied by an increase in caspase activity. Caspase $3 / 7$ activity was inhibited by $33 \%$ in NcaAMPK cells relative to control GFP cells (Figure $3 b$ ). CcaAMPK did not inhibit caspase 3/7 compared with controls (Figure $3 b$ ).

\section{NcaAMPK increases Akt and eNOS phosphorylation}

Previous studies, including ours, suggested that AMPK upregulates the $\mathrm{PKB} /$ Akt signal, ${ }^{9,20}$ which is known to be an important regulator of cell survival in endothelial cells. Consistent with these reports, we found that Akt Ser473 was more highly phosphorylated in NcaAMPKoverexpressing cells than in controls (Figure 4a). Furthermore, eNOS 

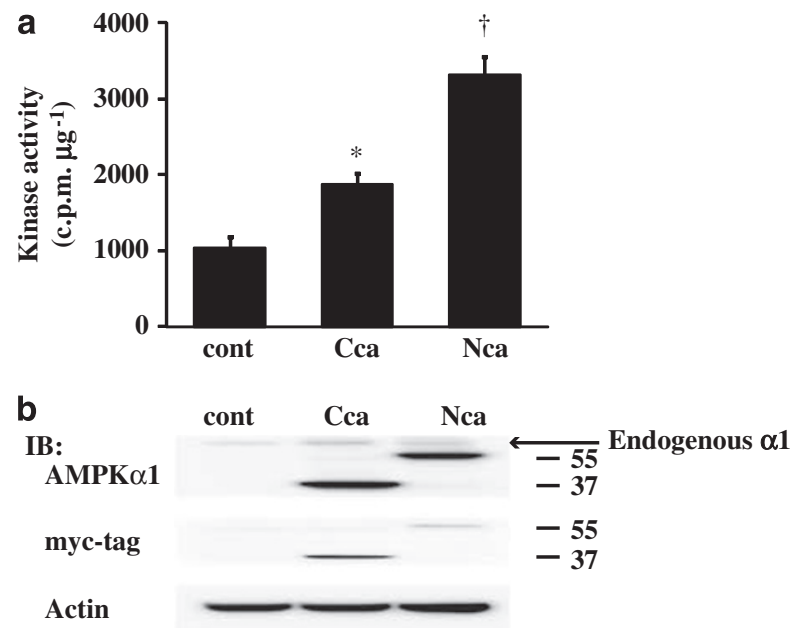

C

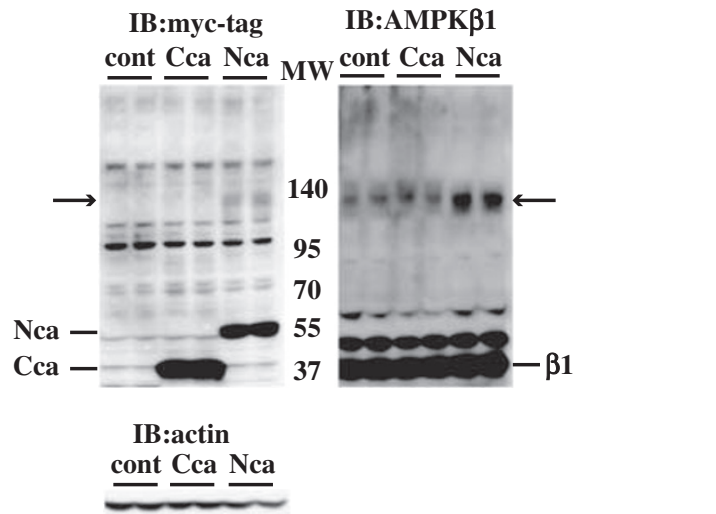

Figure 2 The kinase activity of CcaAMPK and NcaAMPK. (a) Cell lysates of control (cont), CcaAMPK-overexpressing (Cca), or NcaAMPK-overexpressing (Nca) HUVECs were subjected to an AMPK kinase assay using SAMS peptide and $\gamma^{32}$ P-ATP. The activity of Nca was significantly higher than that of cont or Cca. ${ }^{*} P<0.05$ vs. cont, ${ }^{\dagger} P<0.01$ vs. Cca. (b) Western blots using the cell lysates from the experiment shown in panel a. Overexpression of CcaAMPK or NcaAMPK was estimated by using anti-AMPK $\alpha 1$ and anti-myctag antibodies. Actin was blotted as an internal control. (c) Western blots using a UV light-sensitive photoactivated amino acid system. In NcaAMPKoverexpressing cells, positive bands of $\sim 130 \mathrm{kDa}$ were found when using the anti-myc-tag and anti-AMPK $\beta$ antibodies (indicated by arrows). Myc-tagpositive bands of $\sim 110 \mathrm{kDa}$ were not found in CcaAMPK-overexpressing cells. These results suggest that NcaAMPK has higher kinase activity than CcaAMPK because it forms a tighter and more effective AMPK complex. IB, immuno blot.

Ser1177, which is known to be phosphorylated by Akt and AMPK, ${ }^{21}$ was also phosphorylated (Figure 4a). Phosphoinositide 3 (PI3) kinase is an upstream kinase of Akt, and pretreatment with the PI3 kinase inhibitor wortmannin $\left(1 \mu \mathrm{moll} \mathrm{l}^{-1}\right)$ inhibited Akt and eNOS phosphorylation (Figures $4 \mathrm{a}$ and $\mathrm{b}$ ), suggesting that NcaAMPK phosphorylates Akt and eNOS in a PI3 kinase-dependent manner. Thus, eNOS might be phosphorylated both directly by AMPK and, at least in part, by the PI3-Akt pathway.

As we have previously shown that nitric oxide has an anti-apoptotic effect in endothelial cells, ${ }^{22}$ we used the WST-1 assay to investigate whether the nitric oxide synthase inhibitor L-NAME could inhibit the anti-apoptotic cell survival effect of NcaAMPK. Although we used L-NAME concentrations from 1.0 to $5.0 \mathrm{mmoll}^{-1}$, L-NAME pretreatment did not inhibit cell survival (Figure 4c).
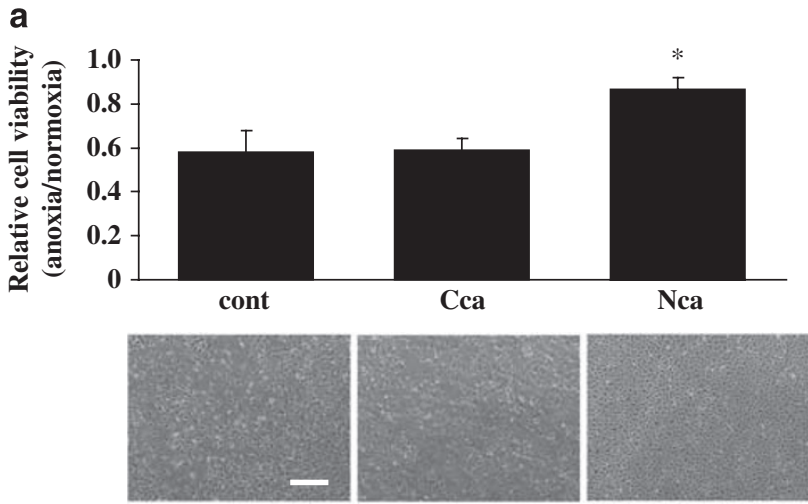

b

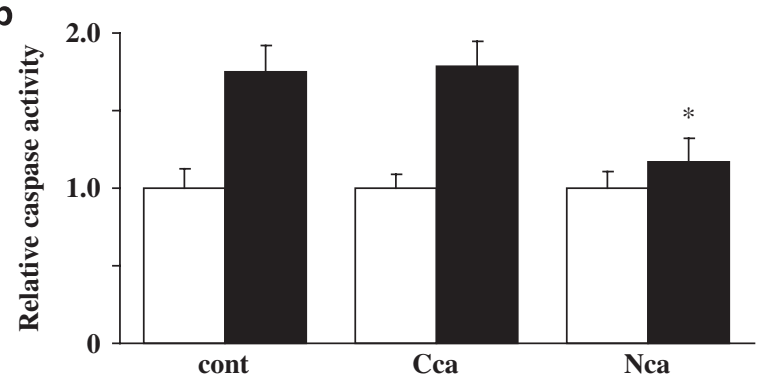

Figure 3 NcaAMPK overexpression inhibits anoxia-induced cell death and caspase 3/7 activity. (a) Ratio of cell viability in anoxia to that in normoxia, as determined in the WST-1 assay. NcaAMPK overexpression ameliorated the anoxia-induced cell death ratio compared with CcaAMPK or control (GFP). Lower panels show phase-contrast pictures of HUVECs. Scale bar indicates $200 \mu \mathrm{m}$. (b) In control cells, anoxia increased caspase 3/7 activity. Although CcaAMPK overexpression did not inhibit caspase 3/7 activity, NcaAMPK overexpression significantly inhibited it. Blank bars and filled bars represent normoxia and anoxia, respectively. cont, GFP; Cca, CcaAMPK; and Nca, NcaAMPK. Each bar represents the mean \pm s.e.m., $N=6$. The experiments were performed three times. ${ }^{*} P<0.01$ vs. cont, Cca.

NcaAMPK increases the phosphorylation of Akt but does not modulate proteins of the bcl-2 family

In agreement with the results of our previous report, ${ }^{9}$ the phosphorylation of Akt Ser473 decreased under anoxic conditions in control cells (Figures $5 \mathrm{a}$ and b). However, in NcaAMPK-overexpressing cells, Akt phosphorylation was maintained at a high level even under anoxic conditions. The expression levels of two members of the Bcl-2 family, $\mathrm{Bax}$ and Bcl-xl, did not change in NcaAMPK-overexpressing cells or under anoxic conditions (Figure 5a). Bcl-2 was not detected by western blot analysis.

NcaAMPK suppresses anoxia-induced cell death and PARP cleavage but co-expression of dnAkt abrogates this suppression

We showed that NcaAMPK overexpression augments the resistance of HUVECs to anoxia (Figure 3). However, co-expression of dnAkt partially but significantly abrogated this anoxia resistance in NcaAMPK-overexpressing cells (Figure 6a). Next, we performed western blot analyses to investigate whether PARP cleavage was inhibited more effectively in NcaAMPK-overexpressing cells than in controls. The band intensities of cleaved PARP were higher under anoxic conditions than under normoxic conditions. The band intensity of the cleaved smaller fragment of PARP was lower in NcaAMPKoverexpressing cells than in controls under anoxic conditions (Figure 6b, lane 4). However, overexpression of dnAkt partially but 
a

IB:

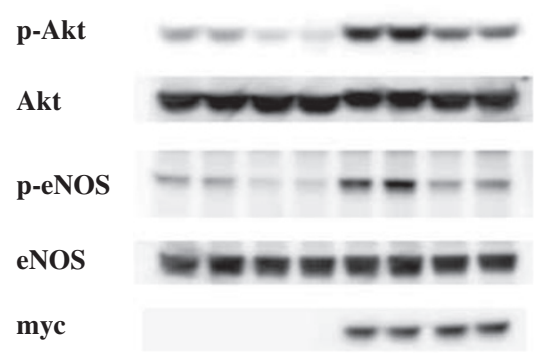

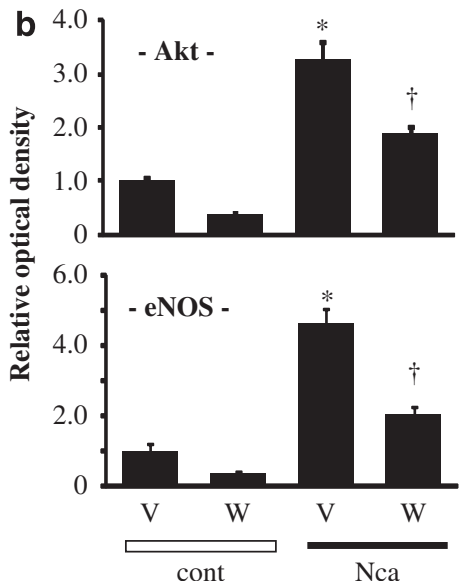

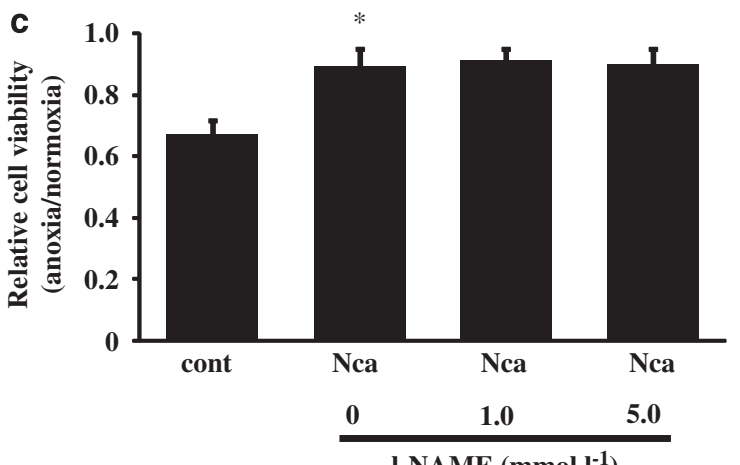

I-NAME ( $\left.\mathrm{mmol} \mathrm{l}^{-1}\right)$

Figure 4 NcaAMPK overexpression increases Akt and eNOS phosphorylation levels. (a) NcaAMPK increases phosphorylation of Akt Ser473 and eNOS Ser1177. The PI3 kinase inhibitor wortmannin inhibited this phosphorylation. V, vehicle; W, wortmannin, cont: GFP, Nca: NcaAMPK. IB: immuno blot. (b) Density levels of Akt Ser473 and eNOS Ser1177 after normalization to the amount of total loaded protein are shown. The mean value of V/cont was fixed to 1.0. Each bar represents the mean \pm s.e.m., $N=4$. ${ }^{*} P<0.005$ vs. cont. ${ }^{\dagger} P<0.01$ vs. V. (c) Cell viability under anoxic conditions relative to that under normoxic conditions as measured using the WST-1 assay. The NOS inhibitor L-NAME did not abrogate the inhibition of anoxia-induced cell death by NcaAMPK. Each bar represents the mean \pm s.e.m., $N=6$. ${ }^{*} P<0.01$ vs. cont.

significantly abrogated the inhibition of PARP cleavage in NcaAMPKoverexpressing cells under anoxic conditions (Figure 6b, lane 8).

\section{DISCUSSION}

The results of this study suggest that AMPK activation by NcaAMPK inhibits anoxia-induced apoptosis in HUVECs through the activation of Akt. Although ischemia and reperfusion have been shown to injure the cardiovascular system, AMPK activation can prevent these harmful effects of ischemia. ${ }^{23}$ AMPK is also a downstream target of adiponectin, ${ }^{24,25}$ the most cardiovascular-protective adipocytokine, ${ }^{26}$ which inhibits cardiomyocyte apoptosis partially through AMPK activation. ${ }^{27}$ Conversely, apoptosis increases in cardiomyocytes after ischemia and reperfusion when AMPK activation is impaired. ${ }^{23}$ Thus, AMPK clearly plays an anti-apoptotic role in the cardiovascular system, although details of the mechanism remain to be elucidated, especially in the vasculature.

Some investigators ${ }^{28,29}$ have shown that AICAR-induced AMPK activation inhibits apoptosis in vascular endothelial cells, whereas other investigators ${ }^{30,31}$ have reported that AICAR treatment, rather than increasing cell viability, actually promotes apoptotic changes in nonendothelial cell lines. AICAR has also been reported to have nonspecific effects in addition to its ability to activate AMPK. ${ }^{12,32,33}$ Therefore, we have been very eager to find a more specific activator of AMPK to clarify whether AMPK signaling actually inhibits anoxiainduced apoptosis in vascular endothelial cells. A constitutively active
AMPK mutant containing only the $\alpha$-subunit kinase domain, CcaAMPK, provided an alternative, but the kinase domain alone possesses weak kinase activity in mammalian cells, and maximum AMPK activity requires all three subunits. ${ }^{6,14}$ We therefore constructed a replication-defective adenovirus expressing NcaAMPK, which has higher kinase activity and suppresses anoxia-induced apoptotic cell death more efficiently than CcaAMPK. Although higher dose, 100 MOI, CcaAMPK transduction in the cells increased SAMS peptide phosphorylation rate by $\sim 35 \%$ compared with $50 \mathrm{MOI}$, the dose we used in this study, we did not find more potent inhibition of cell death in CcaAMPK-overexpressing cells with 100 MOI (data not shown). Our results suggest that the ability of NcaAMPK to bind the $\beta$-subunit might contribute to its more effective suppression of anoxia-induced apoptosis than that of CcaAMPK.

It has been previously reported that Akt is phosphorylated and activated in AMPK-activated endothelial cells, ${ }^{9,20,34}$ and this activation is PI3-kinase dependent. ${ }^{20,34}$ In this study we found that Akt Ser473 was also phosphorylated in NcaAMPK-overexpressing HUVECs. PI3 kinase inhibition by wortmannin suppressed this Akt phosphorylation, suggesting that NcaAMPK also upregulates Akt in a PI3 kinasedependent manner. The anti-apoptotic effect of Akt has been proposed to be partially due tophosphorylation of transcription factors of the forkhead box gene, group O (FoxOs) and Bcl-xL/Bcl-2-associated death promoter (Bad). ${ }^{35-37}$ As we reported previously that phosphorylation levels of Akt were gradually downregulated under hypoxic 

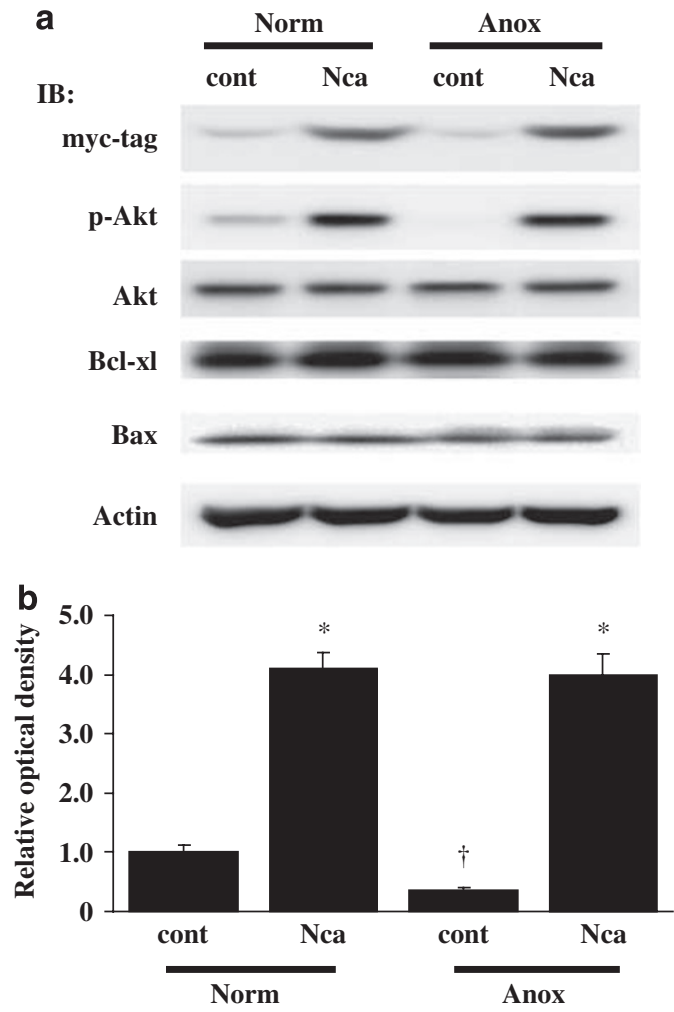

Figure 5 NcaAMPK increases phosphorylation levels of Akt under both normoxic and anoxic conditions. Basal phosphorylation levels of Akt in controls were downregulated under anoxic conditions compared with normoxic conditions. However, in NcaAMPK-overexpressing cells, Akt phosphorylation was maintained at high levels even under anoxic conditions. Neither Bcl-xl nor Bax expression levels changed under anoxic conditions. The experiments were performed four times and a representative figure is shown. IB, immunoblot. (b) Relative phosphorylation levels of Akt were quantified using the Scion Image program. Immunoblots were normalized to total loaded protein. The mean value of cont/Norm (normoxia) was fixed to 1.0. Each bar represents the mean \pm s.e.m., ${ }^{*} P<0.005$ vs. cont, ${ }^{\dagger} P<0.01$ vs. Norm.

conditions, ${ }^{9}$ we also found in this study that the basal phosphorylation levels of Akt were lower under anoxic conditions than in normoxic conditions (Figure 5). However, phosphorylation was maintained at high levels in NcaAMPK-overexpressing cells even under anoxic conditions. As overexpression of dnAkt did not reverse completely the inhibition of anoxia-induced PARP cleavage in NcaAMPK-overexpressing HUVECs, we suggest that NcaAMPK inhibits apoptosis of endothelial cells not only through Akt signaling but also through other, as yet unknown, signaling pathways under anoxic conditions.

Very recently, Young ${ }^{11}$ published a thought-provoking review on the function of AMPK in the cardiovascular system under ischemic stress. He suggested that AMPK functions as a 'major conductor of the stress signaling orchestra' in ischemic cardiovascular cells. As AMPK functions as an energy sensor and inhibits ATP-consuming reactions in endothelial cells, improvement of intracellular energy status might be a major factor for preventing cell death under anoxic/hypoxic conditions. However, we revealed in this study that AMPK activation plays an anti-apoptotic role in HUVECs, at least partly through the Akt pathway under anoxic conditions. AMPK might therefore play a beneficial role in the ischemic vasculature, although the details of the mechanism remain to be elucidated.

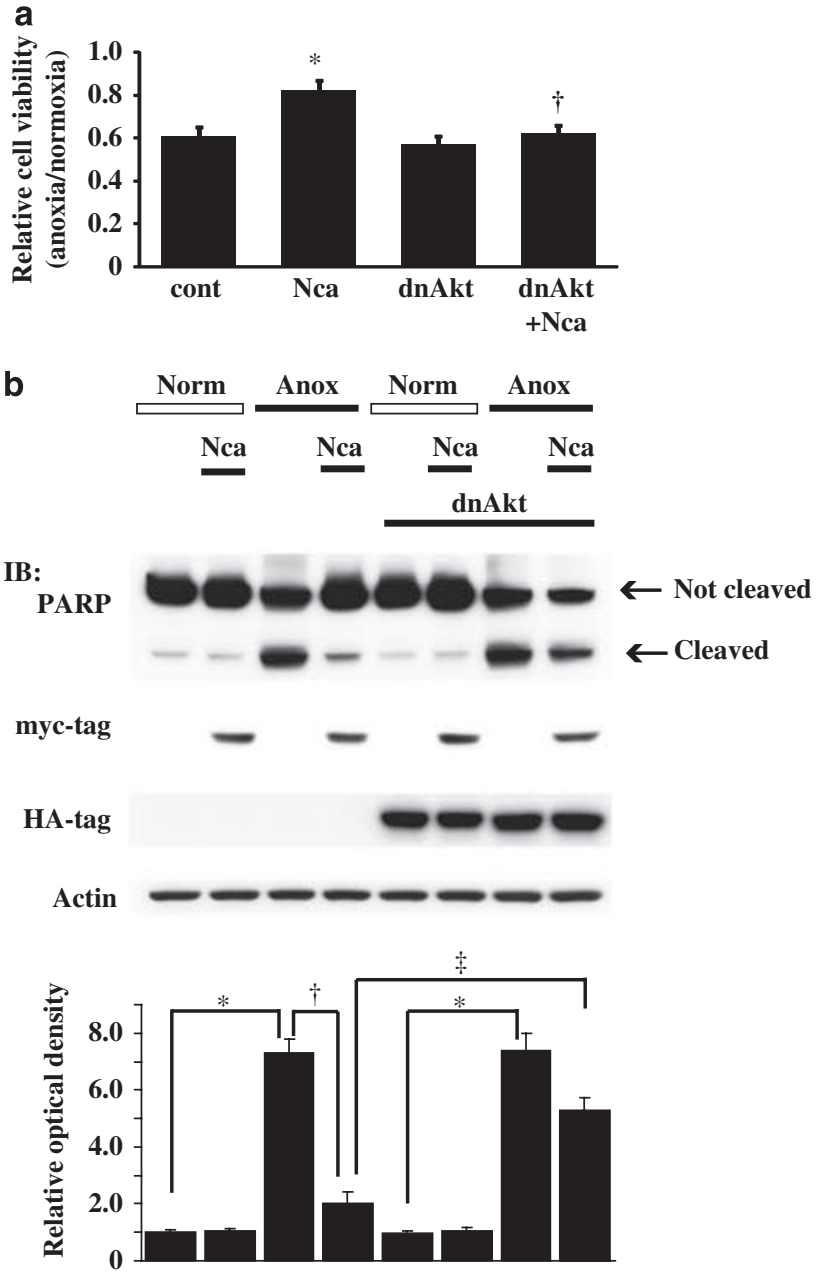

Figure 6 NcaAMPK inhibits anoxia-induced cell death and PARP cleavage but dominant-negative (dn) Akt abrogates this inhibition. (a) The cell viability measured in the WST-1 assay was compared under anoxic conditions among the cells expressing GFP $100 \mathrm{MOI}$ (cont), NcaAMPK $50 \mathrm{MOI}+\mathrm{GFP} 50 \mathrm{MOI}$ (Nca), GFP $50 \mathrm{MOI}+d n A k t 50 \mathrm{MOI}$ (dnAkt), and NcaAMPK $50 \mathrm{MOI}+d n A k t$ $50 \mathrm{MOI}$ (dnAkt+Nca). Each bar represents the mean \pm s.e.m., $N=6$. ${ }^{*} P<0.01$ vs. cont, ${ }^{\dagger} P<0.01$ vs. Nca. (b) Anoxia-induced caspase activation increased PARP cleavage in control HUVECs, as shown with an anti-PARP antibody, and NcaAMPK overexpression inhibited PARP cleavage compared with control. However, co-overexpression of dnAkt abrogated the inhibition of PARP cleavage in NcaAMPK-overexpressing cells under anoxic conditions. Immunoblotting with myc-tag or HA tag antibodies shows the expressions of NcaAMPK and dnAkt, respectively. The experiments were performed four times and a representative figure is shown. The lower part of this figure shows a quantitative analysis of the cleaved PARP fragments. Each bar represents the mean \pm s.e.m., Norm, normoxia; Anox, anoxia; Nca, NcaAMPK; IB, immunoblot. ${ }^{*} P<0.005,{ }^{\dagger} P<0.01,{ }^{\ddagger} P<0.05$.

\section{ACKNOWLEDGEMENTS}

We gratefully acknowledge the excellent technical support of Ms Asuka Ishii, Ms Marie Morita, and Ms Etsuko Kanaya. This study was supported by Grantsin-Aid no. 19590855 (to DN) and no. 17659229 (to YH) and by Core Research for Evolutional Science and Technology (to YH and TN) from the Ministry of Education, Culture, Sports, Science, and Technology of Japan.

1 Ross R. Atherosclerosis - an inflammatory disease. N Engl J Med 1999; 340: 115-126. 
2 Walshe TE, D'Amore PA. The role of hypoxia in vascular injury and repair. Annu Rev Pathol 2008; 3: 615-643.

3 Eefting F, Rensing B, Wigman J, Pannekoek WJ, Liu WM, Cramer MJ, Lips DJ, Doevendans PA. Role of apoptosis in reperfusion injury. Cardiovasc Res 2004; 61: 414-426.

4 Hardie DG, Carling D, Carlson M. The AMP-activated/SNF1 protein kinase subfamily: metabolic sensors of the eukaryotic cell? Annu Rev Biochem 1998; 67: 821-855.

5 Woods A, Munday MR, Scott J, Yang X, Carlson M, Carling D. Yeast SNF1 is functionally related to mammalian AMP-activated protein kinase and regulates acetyl-CoA carboxylase in vivo. J Biol Chem 1994; 269: 19509-19515.

6 Dyck JR, Gao G, Widmer J, Stapleton D, Fernandez CS, Kemp BE, Witters LA. Regulation of $5^{\prime}$-AMP-activated protein kinase activity by the noncatalytic beta and gamma subunits. J Biol Chem 1996; 271: 17798-17803.

7 Cheung PC, Salt IP, Davies SP, Hardie DG, Carling D. Characterization of AMP-activated protein kinase gamma-subunit isoforms and their role in AMP binding. Biochem J 2000; 346: 659-669.

8 Xing Y, Musi N, Fujii N, Zou L, Luptak I, Hirshman MF, Goodyear LJ, Tian R. Glucose metabolism and energy homeostasis in mouse hearts overexpressing dominant negative alpha2 subunit of AMP-activated protein kinase. J Biol Chem 2003; 278 28372-28377.

9 Nagata D, Mogi M, Walsh K. AMP-activated protein kinase (AMPK) signaling in endothelial cells is essential for angiogenesis in response to hypoxic stress. J Biol Chem 2003; 278: 31000-31006.

10 Hong SP, Leiper FC, Woods A, Carling D, Carlson M. Activation of yeast Snf1 and mammalian AMP-activated protein kinase by upstream kinases. Proc Natl Acad Sci USA 2003; 100: 8839-8843.

11 Young LH. AMP-activated protein kinase conducts the ischemic stress response orchestra. Circulation 2008; 117: 832-840.

12 Corton JM, Gillespie JG, Hawley SA, Hardie DG. 5-Aminoimidazole-4-carboxamide ribonucleoside. A specific method for activating AMP-activated protein kinase in intact cells? Eur J Biochem 1995; 229: 558-565.

13 Nagata D, Takeda R, Sata M, Satonaka H, Suzuki E, Nagano T, Hirata Y. AMP-activated protein kinase inhibits angiotensin II-stimulated vascular smooth muscle cell proliferation. Circulation 2004; 110: 444-451.

14 Crute BE, Seefeld K, Gamble J, Kemp BE, Witters LA. Functional domains of the alpha1 catalytic subunit of the AMP-activated protein kinase. J Biol Chem 1998; 273: 35347-35354.

15 Woods A, Azzout-Marniche D, Foretz M, Stein SC, Lemarchand P, Ferré P, Foufelle F, Carling D. Characterization of the role of AMP-activated protein kinase in the regulation of glucose-activated gene expression using constitutively active and dominant negative forms of the kinase. Mol Cell Biol 2000; 20: 6704-6711.

16 Arad M, Benson DW, Perez-Atayde AR, McKenna WJ, Sparks EA, Kanter RJ, McGarry K, Seidman JG, Seidman CE. Constitutively active AMP kinase mutations cause glycogen storage disease mimicking hypertrophic cardiomyopathy. J Clin Invest 2002; 109 357-362.

17 Minokoshi Y, Alquier T, Furukawa N, Kim YB, Lee A, Xue B, Mu J, Foufelle F, Ferré P, Birnbaum MJ, Stuck BJ, Kahn BB. AMP-kinase regulates food intake by responding to hormonal and nutrient signals in the hypothalamus. Nature 2004; 428: 569-574.

18 Schmidt MC, McCartney RR. beta-subunits of Snf1 kinase are required for kinase function and substrate definition. EMBO J 2000; 19: 4936-4943.

19 Warden SM, Richardson C, O'Donnell Jr J, Stapleton D, Kemp BE, Witters LA. Posttranslational modifications of the beta- 1 subunit of AMP-activated protein kinase affect enzyme activity and cellular localization. Biochem J 2001; 354: 275-283.

20 Ouchi N, Kobayashi H, Kihara S, Kumada M, Sato K, Inoue T, Funahashi T, Walsh K. Adiponectin stimulates angiogenesis by promoting cross-talk between AMP-activated protein kinase and Akt signaling in endothelial cells. J Biol Chem 2004; 279 : 1304-1309.

21 Chen ZP, Mitchelhill KI, Michell BJ, Stapleton D, Rodriguez-Crespo I, Witters LA, Power DA, Ortiz de Montellano PR, Kemp BE. AMP-activated protein kinase phosphorylation of endothelial NO synthase. FEBS Lett 1999; 443: 285-289.

22 Sata M, Kakoki M, Nagata D, Nishimatsu H, Suzuki E, Aoyagi T, Sugiura S, Kojima H, Nagano T, Kangawa K, Matsuo H, Omata M, Nagai R, Hirata Y. Adrenomedullin and nitric oxide inhibit human endothelial cell apoptosis via a cyclic GMP-independent mechanism. Hypertension 2000; 36: 83-88.

23 Russell III RR, Li J, Coven DL, Pypaert M, Zechner C, Palmeri M, Giordano FJ, Mu J, Birnbaum MJ, Young LH. AMP-activated protein kinase mediates ischemic glucose uptake and prevents postischemic cardiac dysfunction, apoptosis, and injury. J Clin Invest 2004; 114: 495-503.

24 Yamauchi T, Kamon J, Minokoshi Y, Ito Y, Waki H, Uchida S, Yamashita S, Noda M, Kita S, Ueki K, Eto K, Akanuma Y, Froguel P, Foufelle F, Ferre P, Carling D, Kimura S, Nagai $\mathrm{R}$, Kahn BB, Kadowaki T. Adiponectin stimulates glucose utilization and fatty-acid oxidation by activating AMP-activated protein kinase. Nat Med 2002; 8: 1288-1295.

25 Kadowaki T, Yamauchi T, Kubota N, Hara K, Ueki K, Tobe K. Adiponectin and adiponectin receptors in insulin resistance, diabetes, and the metabolic syndrome. J Clin Invest 2006; 116: 1784-1792.

26 Yamauchi T, Kamon J, Waki H, Imai Y, Shimozawa N, Hioki K, Uchida S, Ito $Y$, Takakuwa K, Matsui J, Takata M, Eto K, Terauchi Y, Komeda K, Tsunoda M, Murakami K, Ohnishi Y, Naitoh T, Yamamura K, Ueyama Y, Froguel P, Kimura S, Nagai R, Kadowaki T. Globular adiponectin protected ob/ob mice from diabetes and ApoEdeficient mice from atherosclerosis. J Biol Chem 2003; 278: 2461-2468.

27 Shibata R, Sato K, Pimentel DR, Takemura Y, Kihara S, Ohashi K, Funahashi T, Ouchi $\mathrm{N}$, Walsh K. Adiponectin protects against myocardial ischemia-reperfusion injury through AMPK- and COX-2-dependent mechanisms. Nat Med 2005; 11: 1096-1103.

28 Ido Y, Carling D, Ruderman N. Hyperglycemia-induced apoptosis in human umbilical vein endothelial cells: inhibition by the AMP-activated protein kinase activation. Diabetes 2002; 51: 159-167.

29 Kim JE, Kim YW, Lee IK, Kim JY, Kang YJ, Park SY. AMP-activated protein kinase activation by 5 -aminoimidazole-4-carboxamide-1-beta-D-ribofuranoside (AICAR) inhibits palmitate-induced endothelial cell apoptosis through reactive oxygen species suppression. J Pharmacol Sci 2008; 106: 394-403.

30 Campas C, Lopez JM, Santidrian AF, Barragán M, Bellosillo B, Colomer D, Gil J. Acadesine activates AMPK and induces apoptosis in B-cell chronic lymphocytic leukemia cells but not in T lymphocytes. Blood 2003; 101: 3674-3680.

31 Kefas BA, Cai Y, Ling Z, Heimberg H, Hue L, Pipeleers D, Van de Casteele M. AMPactivated protein kinase can induce apoptosis of insulin-producing MIN6 cells through stimulation of c-Jun-N-terminal kinase. J Mol Endocrinol 2003; 30: 151-161.

32 Sabina RL, Patterson D, Holmes EW. 5-Amino-4-imidazolecarboxamide riboside (Z-riboside) metabolism in eukaryotic cells. J Biol Chem 1985; 260: 6107-6114.

33 Hardie DG, Carling D. The AMP-activated protein kinase - fuel gauge of the mammalian cell? Eur J Biochem 1997; 246: 259-273.

34 Levine YC, Li GK, Michel T. Agonist-modulated regulation of AMP-activated protein kinase (AMPK) in endothelial cells. Evidence for an AMPK $\rightarrow$ Rac $1 \rightarrow A k t \rightarrow$ endothelial nitric-oxide synthase pathway. J Biol Chem 2007; 282: 20351-20364.

35 Datta SR, Dudek H, Tao X, Masters S, Fu H, Gotoh Y, Greenberg ME. Akt phosphorylation of BAD couples survival signals to the cell-intrinsic death machinery. Cell 1997; 91: 231-241.

36 Brunet A, Bonni A, Zigmond MJ, Lin MZ, Juo P, Hu LS, Anderson MJ, Arden KC, Blenis J, Greenberg ME. Akt promotes cell survival by phosphorylating and inhibiting a Forkhead transcription factor. Cell 1999; 96: 857-868.

37 Huang H, Tindall DJ. Dynamic FoxO transcription factors. J Cell Sci 2007; 120: 2479-2487. 